\title{
Weightlifting Shoulder Injuries Presenting to U.S. Emergency Departments: 2000-2030
}

\author{
Authors \\ Kevin Pirruccio, John D. Kelly
}

\begin{abstract}
Affiliation
Orthopaedic Surgery, University of Pennsylvania, Perelman School of Medicine, Philadelphia, United States
\end{abstract}

Key words

trends, projections, NEISS, emergency

accepted 10.05.2019

Bibliography

DOI https://doi.org/10.1055/a-0927-6818

Int J Sports Med 2019; 40: 528-534

(c) Georg Thieme Verlag KG Stuttgart · New York

ISSN 0172-4622

\author{
Correspondence \\ Mr. Kevin Pirruccio \\ Orthopaedic Surgery, \\ University of Pennsylvania, Perelman School of Medicine, \\ 3400 Spruce Street, \\ 19104 Philadelphia, \\ United States \\ Tel.: + 1/860/992 2265, Fax: + 1/215/829 2492 \\ kevin.pirruccio@pennmedicine.upenn.edu
}

\begin{abstract}
Weightlifting is associated with a significant risk of shoulder injury. We used the National Electronic Injury Surveillance System (NEISS) database to identify patients presenting to U.S. emergency departments between 2000 and 2017 with weightlifting-associated shoulder injuries to determine how the health burden and demographic characteristics of these patients have changed over time. Our analyses demonstrated a significant increase in the national estimate of weightlifting-associated shoulder injuries between 2000 ( $N=8.073$; C.I. 6.309-9.836) and $2017(\mathrm{~N}=14.612$; C.I. 12.293-16.930) $(\mathrm{p}<0.001)$, with linear regression $\left(R^{2}=0.87, P<0.001\right)$ projecting 22.691 national cases by 2030 . Patients were most often males $(83.3 \%$; C.I. 81.5-85.2\%) between 20-29 years of age (30.5\%; C.I. $28.2 \%-32.8 \%)$ sustaining a sprain, strain, or muscle tear (65.1\%; C.I. 60.4-69.8\%). Additionally, the average age of injury (34.33 years; C.I. 33.43-35.23 years) in the 2012-2017 period was significantly higher than in prior periods $(p<0.001)$. We postulated these findings may reflect older individuals more frequently participating in resistance training than in the past, and considered that contemporary fitness trends such as CrossFit might have higher injury rates. We believe increased awareness of this burden, coupled with focus on injury prevention strategies, could yield substantial national health and cost savings.
\end{abstract}

\section{Introduction}

In recent years, it has become increasingly evident that weightlifting can yield numerous health benefits [1]. In addition to its role in reducing stress, weightlifting has been associated with health-promoting effects such as decreased blood pressure, improved glucose control, maintenance of bone mineral density, and resistance to adipose accumulation [2]. Impressively, weightlifting has also been shown to benefit both older adults and adolescents, two groups for which there was once reluctance in advising participation in resistance training. In older adults, resistance training has been specifically shown to help manage osteoarthritis; in adolescents, improvements in motor skills and more healthful body compositions have similarly been reported $[3,4]$. In part, this has led to a significant increase in its national popularity, with about $45 \%$ of the population having reported that they participated in weightlifting in 2011 [5].
Nevertheless, weightlifting carries with it a significant risk of injury that should not be overlooked [6]. In general, injuries can be categorized as musculoskeletal, neurologic, or cardiovascular; they are often associated with poor technique, fatigue, or an overzealous program of training [7]. Injuries most commonly occur in younger adult males secondary to losing control of free weights during exercise $[6,8]$. Often times, the result is a sprain or strain, though more serious injuries - including fractures and dislocations - are regularly observed [6].

The most frequently injured anatomical region associated with weightlifting is the shoulder [9]. This is due in part to its impressive range of motion, which makes it more susceptible to instability [10]. In addition, high-intensity weightlifting forces the normally nonweight bearing glenohumeral joint to endure a significant load, which can result in stress fractures, rotator cuff or labral injury, early cartilage wear, or muscle strain [11]. However, the epidemiology of shoulder injuries associated with weightlifting in the United States, 
and how trends have evolved over time with shifting exercise habits and training methodologies, is poorly characterized.

The purpose of this study is to estimate the national burden and demographic characteristics of patients presenting to emergency departments in the United States between 2000 and 2017 with shoulder injuries related to weightlifting. We then used this data to predict the estimated national burden of weightlifting-associated shoulder injuries between 2017 and 2030. We hypothesized that the total incidence of weightlifting injuries of the shoulder has increased significantly over time as the popularity of weightlifting has continued to rise.

\section{Materials and Methods}

\section{Data sources}

This study used the publicly available, deidentified Consumer Product Safety Commission's (CPSC) National Electronic Injury Surveillance System (NEISS) to perform a retrospective, cross-sectional descriptive epidemiological study identifying cases of shoulder injuries associated with weightlifting (Product Code: 3265; "Weight Lifting (Activity, Apparel, or Equipment”). The CPSC webpage provides publicly available data collection methodologies and general information pertaining to this nationally representative probability sample of hospital emergency departments, which is further stratified by both hospital size and geographic location [12-14]. In this database, data from about 100 designated hospital emergency departments is entered daily; this sample serves as a probability sample of all U.S. hospitals with emergency departments from which weighted national estimates may be derived.

Variables included in the NEISS database include: the date of treatment; the case record number; the age, gender, race and ethnicity of the patient; the injury diagnosis; the body part affected by the injury; disposition (treated and released, admitted, etc.); the product involved (if any) in the injury; the location where the injury occurred; whether fire or motor vehicles were involved in the injury; whether the injury was work-related; whether the injury was intentionally inflicted; a narrative of the incident and scenario leading to the injury.

In this study, each yearly sample in the NEISS database was queried between 2000 and 2017 for all injuries treated in the emergency department. A total of 6678317 unique sample cases were identified, amounting to a weighted national estimate of 245885512 cases during this period. Of these, we specifically identified all injuries of the shoulder associated with weightlifting; 5609 unique cases were observed in the database during this time, yielding a total weighted estimate of 217201 national cases presenting to U.S. emergency departments.

National estimates, standard errors, and $95 \%$ confidence intervals were derived using the svyset function in Stata/IC 15.1, analyzing data in one-year intervals [15] Significance of trends in the total national survey estimates were determined using adjusted Wald tests. P values $<0.05$ (two-sided) were considered significant.

\section{Sources of funding}

This study received no external funding support, and was exempt from IRB review given the use of publicly available, deidentified data from a government website. We also confirm that we have read and understood the International Journal of Sports Medicine ethical standards document, and that our study meets the ethical standards of the journal [16].

\section{Results}

- Table 1 demonstrates a significant increase in the national estimate of shoulder injuries associated with weightlifting presenting to United States emergency departments between $2000(\mathrm{~N}=8073$; C.I. 6309-9 836) and 2017 ( $N=14$ 612; C.I. 12 293-16 930) $(p<0.001)$. $>$ Table 2 further demonstrates that this increase occurred at a faster rate than a parallel but less substantial rise in the total number of injuries due to any cause presenting to U.S. emergency departments. Specifically, the percentage of these total visits that were due to a weightlifting-associated shoulder injury rose significantly between 2000 (0.062\%; C.I. $0.049-0.076 \%)$ and 2017 (0.099\%; C.I. 0.083-0.115\%) ( $p<0.001)$. A linear regression model $\left(R^{2}=0.87, P<0.001\right)$ was used in $>$ Fig. 1 to illustrate this gradual increase over time, and extended to project 22691 shoulder injuries associated with weightlifting presenting to emergency departments in the United States by the year 2030.

The overall demographic characteristics of patients presenting to U.S. emergency departments weightlifting-associated shoulder injuries are detailed in $>$ Table 3 . Nearly two-thirds of presenting patients sustained a sprain or strain, which included muscle tears (65.1\%; C.I. 60.4-69.8\%). Another 16.6\% (C.I. 11.5-21.6\%) were diagnosed with pain or general injury, while $9.3 \%$ (C.I. 7.6-11.0\%) experienced a shoulder dislocation and $0.7 \%$ (C.I. 0.4-1.1\%) a shoulder fracture. Furthermore, the majority of injuries occurred in those 10-19 years of age (19.2\%; C.I. 17.1-21.3\%), 20 to 29 years of age (30.5\%; C.I. $28.2-32.8 \%$ ), and $30-39$ years of age

- Table 1 Weighted national estimates of shoulder injuries associated with weightlifting presenting to U.S. emergency departments, 2000-2017.

\begin{tabular}{|c|l|l|l|l|c|}
\hline Year & $\begin{array}{l}\text { National weighted } \\
\text { estimate of cases }\end{array}$ & $\begin{array}{l}\text { Standard } \\
\text { error }\end{array}$ & \multicolumn{4}{|l|}{$95 \%$ Confidence Interval } \\
\hline 2017 & 14612 & 1167 & 12293 & - & 16930 \\
\hline 2016 & 16311 & 1567 & 13197 & - & 19425 \\
\hline 2015 & 15553 & 1505 & 12563 & - & 18543 \\
\hline 2014 & 14565 & 1222 & 12137 & - & 16993 \\
\hline 2013 & 14963 & 1341 & 12298 & - & 17627 \\
\hline 2012 & 14911 & 950 & 13023 & - & 16799 \\
\hline 2011 & 13739 & 1375 & 11006 & - & 16471 \\
\hline 2010 & 14024 & 1217 & 11606 & - & 16442 \\
\hline 2009 & 13359 & 1401 & 10576 & - & 16142 \\
\hline 2008 & 10195 & 896 & 8415 & - & 11974 \\
\hline 2007 & 11080 & 863 & 9365 & - & 12795 \\
\hline 2006 & 10083 & 839 & 8416 & - & 11750 \\
\hline 2005 & 8014 & 801 & 6422 & - & 9606 \\
\hline 2004 & 9046 & 729 & 7598 & - & 10494 \\
\hline 2003 & 9933 & 996 & 7954 & - & 11912 \\
\hline 2002 & 9606 & 1082 & 7456 & - & 11755 \\
\hline 2001 & 9137 & 995 & 7161 & - & 11113 \\
\hline 2000 & 8073 & 888 & 6309 & - & 9836 \\
\hline & & & & & \\
\hline
\end{tabular}


- Table 2 Percentage of the total number of injuries presenting to U.S. emergency departments due to shoulder injuries associated with weightlifting, 2000-2017.

\begin{tabular}{|c|c|c|c|c|c|c|}
\hline Year & $\begin{array}{l}\text { National weighted estimate of U.S. } \\
\text { ED visits due to weightlifting- } \\
\text { associated shoulder injuries }\end{array}$ & $\begin{array}{l}\text { National weighted estimate of } \\
\text { all injuries treated in U.S. EDs } \\
\text { due to any cause }\end{array}$ & $\begin{array}{l}\text { Percentage of U.S. ED visits due } \\
\text { to weightlifting-associated } \\
\text { shoulder injuries }\end{array}$ & \multicolumn{3}{|c|}{$95 \%$ Confidence Interval } \\
\hline 2017 & 14612 & 14727704 & $0.099 \%$ & $0.083 \%$ & - & $0.115 \%$ \\
\hline 2016 & 16311 & 14319418 & $0.114 \%$ & $0.092 \%$ & - & $0.136 \%$ \\
\hline 2015 & 15553 & 14132697 & $0.110 \%$ & $0.089 \%$ & - & $0.131 \%$ \\
\hline 2014 & 14565 & 13860971 & $0.105 \%$ & $0.088 \%$ & - & $0.123 \%$ \\
\hline 2013 & 14963 & 14033745 & $0.107 \%$ & $0.088 \%$ & - & $0.126 \%$ \\
\hline 2012 & 14911 & 14614128 & $0.102 \%$ & $0.089 \%$ & - & $0.115 \%$ \\
\hline 2011 & 13739 & 14162084 & $0.097 \%$ & $0.078 \%$ & - & $0.116 \%$ \\
\hline 2010 & 14024 & 14694928 & $0.095 \%$ & $0.079 \%$ & - & $0.112 \%$ \\
\hline 2009 & 13359 & 13966898 & $0.096 \%$ & $0.076 \%$ & - & $0.116 \%$ \\
\hline 2008 & 10195 & 13456353 & $0.076 \%$ & $0.063 \%$ & - & $0.089 \%$ \\
\hline 2007 & 11080 & 13232338 & $0.084 \%$ & $0.071 \%$ & - & $0.097 \%$ \\
\hline 2006 & 10083 & 13232263 & $0.076 \%$ & $0.064 \%$ & - & $0.089 \%$ \\
\hline 2005 & 8014 & 12608693 & $0.064 \%$ & $0.051 \%$ & - & $0.076 \%$ \\
\hline 2004 & 9046 & 13096983 & $0.069 \%$ & $0.058 \%$ & - & $0.080 \%$ \\
\hline 2003 & 9933 & 12720947 & $0.078 \%$ & $0.063 \%$ & - & $0.094 \%$ \\
\hline 2002 & 9606 & 12790786 & $0.075 \%$ & $0.058 \%$ & - & $0.092 \%$ \\
\hline 2001 & 9137 & 13310088 & $0.069 \%$ & $0.054 \%$ & - & $0.083 \%$ \\
\hline 2000 & 8073 & 12924488 & $0.062 \%$ & $0.049 \%$ & - & $0.076 \%$ \\
\hline
\end{tabular}

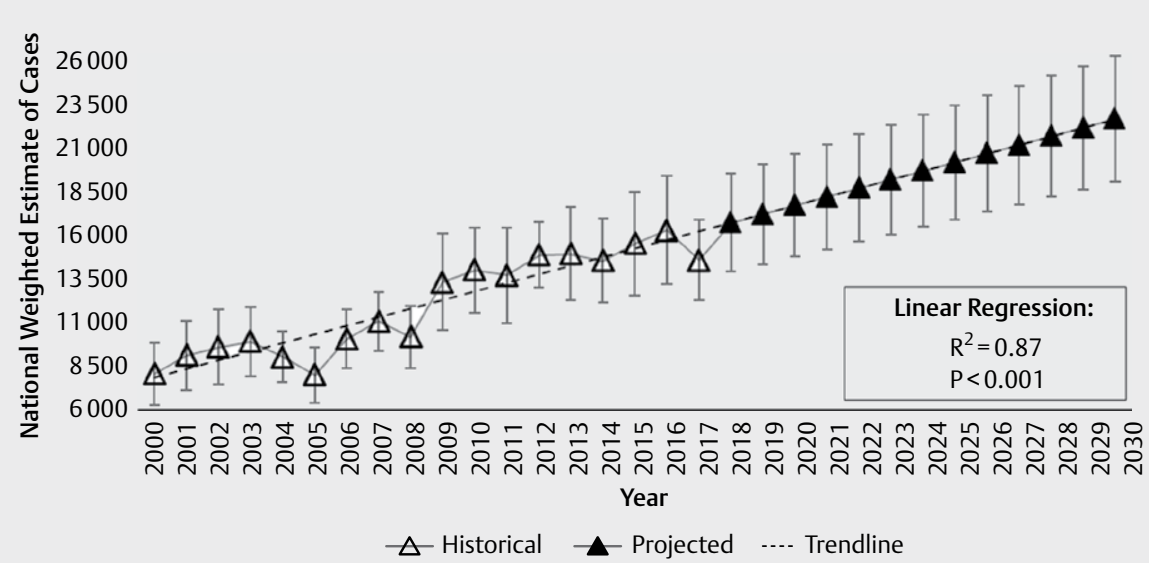

-Fig. 1 This figure demonstrates the historical (outlined, unfilled triangles) and projected (filled black triangles) weighted national estimates of shoulder injuries associated with weightlifting presenting to U.S. emergency departments, 2000-2030. Furthermore, the increased trend in injuries across years is demonstrated using a linear regression model $(R 2=0.87, P<0.001)$. The regression shows that the national estimate of weightliftingassociated shoulder injuries has been increasing gradually across time, at a rate of approximately 500 additional cases per year presenting to U.S. emergency departments. The regression model is then extended to the year 2030, to demonstrate that the national estimate of cases would approach twenty-five thousand cases per year by this time period.

(21.3\%; C.I. 19.9-22.7\%). Patients were most commonly white (45.8\%; C.I. 37.6-53.9\%) and male (83.3\%; C.I. 81.5-85.2\%), and nearly all were treated and released (98.6\%; C.I. 98.0-99.2\%) from the emergency department. Though $39.7 \%$ (C.I. 33.4-46.0\%) of injuries occurred in an unknown location, 36.5\% (C.I. 32.0-40.9\%) occurred while participating in a sports setting, such as at a gym. - Fig. 2 further shows that the percentage of weightliftingassociated shoulder injuries in school $(p=0.45)$ or unknown locations $(p=0.73$ ) has not changed significantly across time. However, the percentage of these injuries occurring at home has decreased significantly $(p<0.001)$ between the 2000-2005 period (21.5\%; C.I. 15.5-27.5\%) and the 2012-2017 period (9.9\%; C.I. $6.8-13.0 \%$ ); the percentage said injuries occurring in sports settings has correspondingly increased significantly from the 2000 2005 period (26.0\%; C.I. 20.3-31.6\%) to the 2012-2017 period (42.7\%; C.I. 36.3-49.1\%). 
> Table 3 Overall demographics of patients presenting to U.S. emergency departments with shoulder injuries associated with weightlifting, $2000-2017$.

\begin{tabular}{|c|c|c|c|c|c|c|}
\hline \multirow{2}{*}{$\begin{array}{l}\text { Demographic variable } \\
\text { Diagnosis }\end{array}$} & \multirow[t]{2}{*}{ National weighted estimates } & \multirow[t]{2}{*}{ Percentage * } & \multirow[t]{2}{*}{ SE } & \multicolumn{3}{|c|}{ 95\% Confidence Interval } \\
\hline & & & & & & \\
\hline Sprain or strain (including tears) & 141364 & $65.1 \%$ & $2.4 \%$ & $60.4 \%$ & - & $69.8 \%$ \\
\hline Pain or general injury & 36015 & $16.6 \%$ & $2.6 \%$ & $11.5 \%$ & - & $21.6 \%$ \\
\hline Dislocation & 20138 & $9.3 \%$ & $0.8 \%$ & $7.6 \%$ & - & $11.0 \%$ \\
\hline Fracture & 1577 & $0.7 \%$ & $0.2 \%$ & $0.4 \%$ & - & $1.1 \%$ \\
\hline Contusions & 3984 & $1.8 \%$ & $0.3 \%$ & $1.3 \%$ & - & $2.4 \%$ \\
\hline Other & 14122 & $6.5 \%$ & $0.8 \%$ & $4.9 \%$ & - & $8.1 \%$ \\
\hline \multicolumn{7}{|l|}{ Age } \\
\hline $0-9 \mathrm{yrs}^{\mathrm{a}}$ & 276 & $0.1 \%$ & & & & \\
\hline $10-19$ yrs & 41656 & $19.2 \%$ & $1.0 \%$ & $17.1 \%$ & - & $21.3 \%$ \\
\hline $20-29$ yrs & 66248 & $30.5 \%$ & $1.2 \%$ & $28.2 \%$ & - & $32.8 \%$ \\
\hline $30-39$ yrs & 46242 & $21.3 \%$ & $0.7 \%$ & $19.9 \%$ & - & $22.7 \%$ \\
\hline $40-49$ yrs & 34727 & $16.0 \%$ & $1.0 \%$ & $14.1 \%$ & - & $17.9 \%$ \\
\hline $50-59$ yrs & 17217 & $7.9 \%$ & $0.6 \%$ & $6.8 \%$ & - & $9.1 \%$ \\
\hline $60-69$ yrs & 6868 & $3.2 \%$ & $0.3 \%$ & $2.6 \%$ & - & $3.8 \%$ \\
\hline $70-79$ yrs & 3444 & $1.6 \%$ & $0.3 \%$ & $1.0 \%$ & - & $2.2 \%$ \\
\hline 80 years or oldera & 524 & $0.2 \%$ & & & & \\
\hline \multicolumn{7}{|l|}{ Sex } \\
\hline Male & 180998 & $83.3 \%$ & $0.9 \%$ & $81.5 \%$ & - & $85.2 \%$ \\
\hline Female & 36132 & $16.6 \%$ & $0.9 \%$ & $14.8 \%$ & - & $18.5 \%$ \\
\hline \multicolumn{7}{|l|}{ Race } \\
\hline White & 99421 & $45.8 \%$ & $4.1 \%$ & $37.6 \%$ & - & $53.9 \%$ \\
\hline Black & 36555 & $16.8 \%$ & $2.9 \%$ & $11.0 \%$ & - & $22.6 \%$ \\
\hline Other & 4066 & $1.9 \%$ & $0.6 \%$ & $0.7 \%$ & - & $3.1 \%$ \\
\hline Hispanic & 11815 & $5.4 \%$ & $1.6 \%$ & $2.2 \%$ & - & $8.7 \%$ \\
\hline Race not specified & 63704 & $29.3 \%$ & $4.3 \%$ & $20.8 \%$ & - & $37.9 \%$ \\
\hline Treated and released from ED & 214140 & $98.6 \%$ & $0.3 \%$ & $98.0 \%$ & - & $99.2 \%$ \\
\hline \multicolumn{7}{|l|}{ Location } \\
\hline Unknown & 86168 & $39.7 \%$ & $3.2 \%$ & $33.4 \%$ & - & $46.0 \%$ \\
\hline Home & 32984 & $15.2 \%$ & $2.0 \%$ & $11.3 \%$ & - & $19.1 \%$ \\
\hline School & 11923 & $5.5 \%$ & $0.6 \%$ & $4.2 \%$ & - & $6.8 \%$ \\
\hline Sports & 79178 & $36.5 \%$ & $2.2 \%$ & $32.0 \%$ & - & $40.9 \%$ \\
\hline \multicolumn{7}{|c|}{$\begin{array}{l}\text { Legend: a - The estimate is considered to be potentially unstable due to the number of unweighted cases from the sample frame totaling }<20 \text {, the } \\
\text { weighted national estimate totaling }<1200 \text {, or coefficient of variation }>33 \% \text {. Therefore, no standard errors or confidence intervals are provided; the } \\
\text { unstable percentage estimate is provided for reference purposes only, as is the weighted national estimate. Omitting certain variables with case } \\
\text { totals }<20 \text { and }<1 \text { significant figure resulted in some totals }<100 \% ;{ }^{*} \text { - Percentages are based on analyses of weighted national estimates (reported } \\
\text { here), which allows for reliable and stable estimates. This is because injury cases have different statistical weights, such that raw numbers from the } \\
\text { sample frame should never be used for comparative purposes; ED - Emergency Department; SE - Standard Error. }\end{array}$} \\
\hline
\end{tabular}

Lastly, $\triangleright$ Fig. 3 demonstrates the average ages of those sustaining shoulder injuries due to weightlifting and presenting to U.S. emergency departments across time, as well as weighted national estimates of these injuries during each period stratified by the following 3 age groups: $\leq 18$ years of age, 19-64 years of age, and $\geq$ 65 years of age. In the 2000-2005 period, the average age of injury was 30.93 years (C.I. 29.85-32.01 years), which was significantly $(p<0.001)$ different from the average age of injury by the 20122017 period (34.33 years; C.I. 33.43-35.23 years). The bar graph further shows that these increases in average age across time for those with weightlifting-associated shoulder injuries is being driven largely by a significantly increasing injury burden in middle-aged patients (19-64 years of age) across periods $(p<0.001)$, and more recent increase $(p<0.01)$ in said injuries by older patients ( $\geq 65$ years of age) between the 2006-2011 and 2012-2017 periods.

\section{Discussion}

Our findings are in accordance with our initial hypothesis that the national number of shoulder injuries associated with weightlifting presenting to United States emergency departments has increased significantly over time. In fact, annual estimates nearly doubled in recent history, rising from over 8,000 injuries per year in 2000 to about 15000 in 2017. At this rate, the incidence of shoulder injury secondary to weightlifting is projected to rise by almost 10000 injuries per year by 2030. Moreover, the data was consistent with 


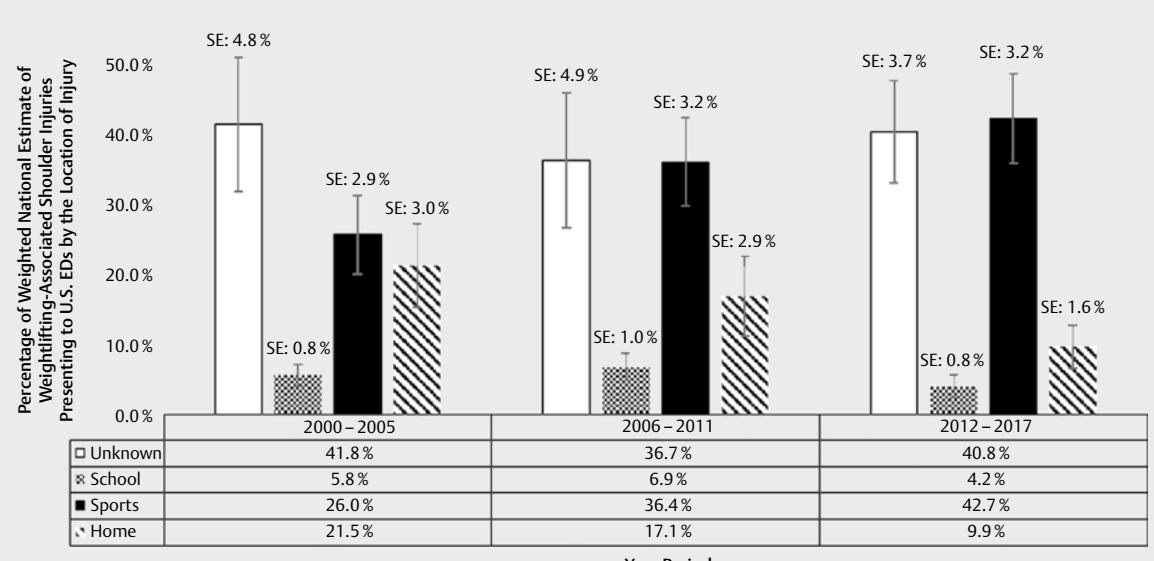

Year Period

Fig. 2 This bar graph shows the percentage of patients in a given year period sustaining a weightlifting-associated shoulder injury and presenting to a U.S. emergency department categorized by the location in which the injury occurred (Unknown Location - Outlined and Unfilled; School Checkered Pattern; Sports - Filled Black; Home - Diagonal Black Stripes). Positive and negative error bars capture the $95 \%$ confidence interval for the derived percentage, based on national weighted estimates of cases and with standard error (SE) labels above said bars. Periods were grouped by six-year intervals for sufficient statistical power. The data table beneath represents the average percentage calculated, by location of injury and year period, corresponding to the graphical values above.

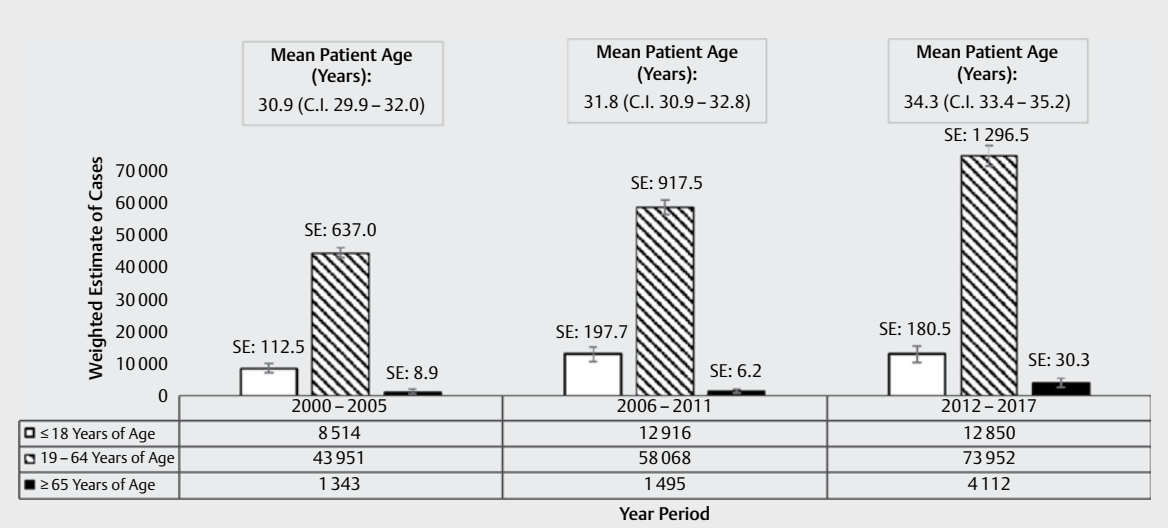

-Fig. 3 This bar graph shows the weighted national estimate of weightlifting-associated shoulder injuries presenting to U.S. emergency departments across time stratified by three separate age groups ( $\leq 18$ years of age - Outlined and Unfilled; 19-64 years of age - Diagonal Black Stripes; $\geq 65$ years of age - Filled Black), grouping periods by six-year intervals for sufficient statistical power. Positive and negative error bars capture the $95 \%$ confidence interval for the derived weighted estimate, with standard error (SE) labels above said bars. The data table beneath shows the national weighted estimate of the number of patients presenting to a U.S. emergency department with a weightlifting-associated shoulder injury stratified by these same age groups, and corresponding to the values in the graph above, for a given period. Then, the average ages during each year period (and $95 \%$ confidence intervals for the average age) of patients presenting to U.S. emergency departments with shoulder injuries associated with weightlifting are provided immediately above the columns for a given time period in text boxes.

prior epidemiological studies regarding weightlifting injuries. For example, Kerr et al. similarly queried the NEISS database 1990 to 2007 for weightlifting-associated injuries presenting to United States emergency departments, and found that $82.3 \%$ of patients were male and that the most common diagnosis was a sprain or strain (46.1\%) [6] Our study obtained comparable demographic results, though since our study was specific to shoulder injuries, reflected more sprains and strains (65.1\%). Importantly, we are the first to show that the average ages of patients presenting to U.S. emergency departments with weightlifting-associated shoulder injuries has steadily risen over time.

There are several explanations as to the observed increasing trend in both weightlifting-associated shoulder injuries and ages of injured patients. For example, there may be a greater pool of individuals participating in weightlifting today than in the past. Recent trends in global exercise habits have demonstrated that more and more people are regularly incorporating strength training into their workouts, concordant with emerging evidence that it can yield health benefits for people of all ages $[2,4,17]$. As middle-aged and 
older adults continue to participate in resistance training and increase the pool of at-risk individuals, they may also be raising the average age of injury higher over time, as was observed in the data. This is also possibly explained by many increasingly looking towards resistance training as a way to cope with rising levels of stress in society $[18,19]$. Given that many of these stressors have been identified as occurring in the workplace, this may select for older weightlifting participants aiming to improve their sense of wellbeing $[20,21]$. There may also have been changes in care-seeking behavior by injured individuals over time, prompting more patients to present to U.S. emergency departments with non-urgent injuries resultant from training [22].

In contrast, other contemporary workout programs may carry a higher risk of injury. In particular, the newfound popularity of CrossFit, which has often been criticized for its extremes of exercise methodology and dieting regimens, is frequently implicated in putting individuals at risk for shoulder injuries due to its emphasis on high-intensity overhead movements and proclivity for recruiting untrained participants [23-28]. Group fitness classes have also grown in popularity among older individuals, and may incorporate resistance training [17]. With less individual supervision and instruction by trainers, participants may be at increased risk for injury since fewer opportunities are afforded for correcting improper form $[29,30]$. This serves as another plausible explanation as to why the average age of weightlifting-associated shoulder injuries continues to rise, though the cause for this phenomenon are likely multifactorial.

Prevention strategies must be implemented to reduce the rate of weightlifting-associated shoulder injuries and the resultant national health burden. In most cases, injuries can be largely prevented by using proper exercise form, employing safe practices when using heavy weights, and training a variety of muscle groups to maintain overall soft tissue balance [31]. When injury does occur, a multitude of treatment options can be considered based on the severity of the soft tissue or bone damage. Mild injuries are often managed with conservative treatment, such as allowing time for rest and recovery, using anti-inflammatory medications, and modifying workouts to reduce further injury; if conservative treatment proves ineffective, surgical options including arthroscopy can reduce pain and greatly restore function to the shoulder [11,31].

This study has several limitations related to the nature of the NEISS database. First, the data only reflects injuries that presented to United States emergency departments. Therefore, individuals who sustained injuries but presented first in an urgent care clinic or an alternative outpatient setting were not captured in our study. Thus, we consider our results to reflect conservative estimates of weightlifting-associated shoulder injury, capturing only those severe enough that patients required emergency medical attention to manage their pain or dysfunction. Second, the NEISS database does not provide any cost data, making it impossible to accurately ascertain the national economic burden caused by weightliftingassociated shoulder injuries. Given the variety of injuries and demographic characteristics observed in our study, using cost multipliers would most likely have resulted in an erroneous final cost estimate. In addition, the NEISS database does not provide information on the type of exercise that resulted in the injury. These details may be sparsely provided in the narrative section, but their in- consistent inclusion makes it a poor choice for identifying exercise characteristics associated with specific injuries. This would have allowed for a much more clinically relevant analysis. Finally, longer term sequelae of weightlifting, especially rotator cuff tear and premature glenohumeral arthritis, were not included in the estimation of injury prevalence, although the financial burden these conditions incur is inordinate [32].

Weightlifting has established itself as a staple of health and wellness in recent years. The benefits of resistance training extend from adolescents to older adults, and manifest not only as enhanced physical strength but also improved mental health. Nonetheless, participation in weightlifting carries with it an inherent risk, especially to the shoulder, meriting furthered awareness of this subject given an increasing annual financial and emotional burden. Attention directed towards injury prevention strategies may yield substantial health and cost savings at the national level.

\section{Conflict of Interest}

Author JK declares that he served as a consultant for Flexion Therapeutics and received book royalties from Springer International Publishing in the past 3 years; Author KP declares no conflicts of interest during this same period.

\section{References}

[1] Winett RA, Carpinelli RN. Potential health-related benefits of resistance training. Prev Med 2001; 33: 503-513

[2] Westcott WL. Resistance training is medicine: effects of strength training on health. Curr Sports Med Rep 2012; 11: 209-216

[3] Barbieri D, Zaccagni L. Strength training for children and adolescents: Benefits and risks. Coll Antropol 2013; 37 (Suppl 2): 219-225

[4] Latham N, Liu C]. Strength training in older adults: The benefits for osteoarthritis. Clin Geriatr Med 2010; 26: 445-459

[5] Katzmarzyk PT, Lee IM, Martin CK, Blair SN. Epidemiology of physical activity and exercise training in the united states. Prog Cardiovasc Dis 2017; 60: 3-10

[6] Kerr ZY, Collins CL, Comstock RD. Epidemiology of weight trainingrelated injuries presenting to United States emergency departments, 1990-2007. Am J Sports Med 2010; 38: 765-771

[7] Fred HL. More on weightlifting injuries. Tex Heart Inst ] 2014; 41: 453-454

[8] Chevan J. Demographic determinants of participation in strength training activities among U.S. adults. J Strength Cond Res 2008; 22: 553-558

[9] Kolber M], Beekhuizen KS, Cheng MS, Hellman MA. Shoulder injuries attributed to resistance training: A brief review. J Strength Cond Res 2010; 24: 1696-1704

[10] Kammel KR, Leber EH. Dislocation, Shoulder (Humerus), Posterior. StatPearls. Treasure Island (FL): StatPearls Publishing LLC; 2018

[11] Haupt HA. Upper extremity injuries associated with strength training. Clin Sports Med 2001; 20: 481-490

[12] CPSC US. NEISS, The National Electronic Injury Surveillance System: A Tool for Researchers 2000

[13] Schroeder T, Ault K. The NEISS sample: Design and implementation. Washington, DC: US Consumer Product Safety Commission; 2001 
[14] US Consumer Product Safety Commission. National Electronic Injury Surveillance System (NEISS) Coding Manual. 2017

[15] StataCorp LP. Stata/IC 15.1 for Windows. StataCorp LP; College Station, TX, USA: 2018

[16] Harriss DJ, Macsween A, Atkinson G. Standards for ethics in sport and exercise science research: 2018 update. Int J Sports Med 2017; 38: $1126-1131$

[17] Thompson WR. Worldwide survey of fitness trends for 2015: What's Driving the Market. ACSMs Health Fit J 2014; 18: 8-17

[18] Craft LL, Perna FM. The Benefits of Exercise for the Clinically Depressed. Prim Care Companion J Clin Psychiatry 2004; 6: 104-111

[19] Strickland JC, Smith MA. The anxiolytic effects of resistance exercise. Front Psychol 2014; 5: 753

[20] Bretland R], Thorsteinsson EB. Reducing workplace burnout: The relative benefits of cardiovascular and resistance exercise. Peer J 2015; 3: e891

[21] Maslach C, Leiter MP. Understanding the burnout experience: Recent research and its implications for psychiatry. World Psychiatry 2016; 15: 103-111

[22] McGuigan T, Watson P. Non-urgent attendance at emergency departments. Emerg Nurse 2010; 18: 34-38

[23] Claudino JG, Gabbett T], Bourgeois F, Souza HS, Miranda RC, Mezencio B, Soncin R, Cardoso Filho CA, Bottaro M, Hernandez AJ, Amadio AC, Serrao JC. CrossFit overview: Systematic review and meta-analysis. Sports Med Open 2018; 4: 5
[24] Dawson MC. CrossFit: Fitness cult or reinventive institution? Int Rev Sociol Sport 2017; 52: 361-379

[25] Klimek C, Ashbeck C, Brook A], Durall C. Are injuries more common with crossfit training than other forms of exercise? J Sport Rehabil 2018; 27: 295-299

[26] Montalvo AM, Shaefer H, Rodriguez B, Li T, Epnere K, Myer GD. Retrospective injury epidemiology and risk factors for injury in crossfit. J Sports Sci Med 2017; 16: 53-59

[27] Summitt RJ, Cotton RA, Kays AC, Slaven EJ. Shoulder injuries in individuals who participate in crossfit training. Sports Health 2016; 8: 541-546

[28] Weisenthal BM, Beck CA, Maloney MD, DeHaven KE, Giordano BD. injury rate and patterns among crossfit athletes. Orthop J Sports Med 2014; 2: 2325967114531177

[29] Bratland-Sanda S, Sundgot-Borgen J, Myklebust G. Injuries and musculoskeletal pain among Norwegian group fitness instructors. Eur ] Sport Sci 2015; 15: 784-792

[30] Gray SE, Finch CF. The causes of injuries sustained at fitness facilities presenting to Victorian emergency departments - identifying the main culprits. Inj Epidemiol 2015; 2: 6

[31] Golshani K, Cinque ME, O'Halloran P, Softness K, Keeling L, Macdonell JR. Upper extremity weightlifting injuries: Diagnosis and management. J Orthop 2017; 15: 24-27

[32] George MS. Simultaneous acute rotator cuff tear and distal biceps rupture in a strongman competitor. Orthopedics 2010; 33 https://doi. org/10.3928/01477447-20100225-28 